\title{
Neonatal Endotracheal Tubes and Prevention of Bronchial Intubation
}

\author{
Hipolito RB ${ }^{1}$, Milstein $\mathrm{JM}^{1}$, Sherman $\mathrm{J}^{2,3}$ and Sherman $\mathrm{MP}^{\star 1,2}$ \\ ${ }^{1}$ Department of Pediatrics University of California, Davis, California \\ ${ }^{2}$ Department of Child Health \\ ${ }^{3}$ Sinclair School of Nursing University of Missouri, Columbia, Missouri
}

${ }^{*}$ Corresponding author: Sherman MP, M.D., F.A.A.P., Professor, Women's and Children's Hospital, University of Missouri Health Care, Division of Neonatology, Suite 206, 404 Keene Street, Columbia, Missouri 65201, Fax: (573) 884-1795, Tel: (573) 882-2272, E-mail: shermanmp@missouri.edu

Citation: Hipolito RB, Milstein JM, Sherman J, Sherman MP (2015) Neonatal Endotracheal Tubes and Prevention of Bronchial Intubation. J Paedatr Neonatal Dis 1(1): 101. doi: 10.15744/2456-5482.1.101

Received Date: April 21, 2015 Accepted Date: June 30, 2015 Published Date: July 01, 2015

\begin{abstract}
Background: Bronchial intubation increases neonatal morbidity. Endotracheal tubes (ETT) are marked to avoid this event. No studies have specifically examined whether the markings on neonatal ETTs reduce right main stem bronchial intubation (RMSBI).

Objectives: The major research objective was to reduce pulmonary morbidity in extremely preterm infants. In Aim 1, we determined retrospectively the incidence of RMSBI after oro-tracheal placement of ETTs. In Aim 2, we conducted a prospective, randomized clinical trial to ascertain whether a new endotracheal tube design prevented bronchial intubation.

Methods: We reviewed chest radiographs to establish the historical incidence of RMSBI. During a clinical trial, we intubated control infants with an ETT having standard markings compared to newborns intubated with an ETT with proximally placed, colored lines. We placed ETTs in control infants using the $7-8-9 \mathrm{~cm}$ rule to judge the depth of insertion. We reduced the depth of insertion by $0.5 \mathrm{~cm}$ when using the new ETT design because a colored line is located next to the upper gingiva.

Results: The retrospective study showed bronchial intubation occurred in $57 \%$ of infants born at 23 to 26 weeks of gestation compared to an overall $14 \%$ of neonates born after 26 weeks ( $p<.001)$. In the clinical trial of a new ETT design, $26 \%$ (8 of 31$)$ of controls had RMSBI after initial ETT placement or during assisted ventilation, while no infants intubated with the new ETT had this complication initially or during subsequent ventilator care $(p<.001)$.

Conclusions: Bronchial intubation is common in extremely preterm infants intubated with commercially marked ETTs. A new ETT design that locates a proximal colored line to the upper gingiva ensured proper placement in the mid-trachea of neonates.

Keywords: Endotracheal tube; Bronchial intubation; Extremely low birth weight infants; Chest radiograph; Depth of intubation; Endotracheal tube design; Endotracheal tube stabilizing devices
\end{abstract}

\section{Introduction}

Right main stem bronchial intubation (RMSBI) causes morbidity during neonatal assisted ventilation. Over-distention of the right middle and/or lower lobes of the lung and under-ventilation and/or atelectasis of the remaining lung are the major complications of RMSBI [1,2]. Complications of RMSBI include a) over-inflation of a pulmonary lobe (lobar emphysema), b) pulmonary interstitial emphysema, c) pneumothorax, and/or d) pneumomediastinum [3]. In very preterm infants, pneumothorax correlates with the pathophysiology of intraventricular hemorrhage [4].

The first study that published the incidence of bronchial intubation in neonates was over four decades ago [1]. In 1971, infants with birth weights $<1000 \mathrm{~g}$ only occasionally survived compared to today. Because of anatomy, extremely low birth weight (ELBW) infants have a higher risk of RMSBI because the distance from the larynx to carina is reduced compared to larger neonates [5]. Since ELBW infants also need a longer period of assisted ventilation [6], we theorized that the incidence of RMSBI places ELBW infants at a higher risk. We undertook a retrospective study to find out if markings used on ETTs for 30 years had reduced RMSBI in neonates. If they did not, we envisioned a new ETT design that might lower this complication. We also postulated a new endotracheal tube (ETT) design could particularly reduce RMSBI in ELBW infants. To evaluate our hypothesis, we placed circumferential, colored lines proximally on ETTs. Each colored line represented a depth of insertion to the mid-trachea when the colored line was adjacent to the upper gingiva (Figure 1). Based on an initial clinical trial, we report this ETT design lowers the risk of RMSBI in ELBW infants. 


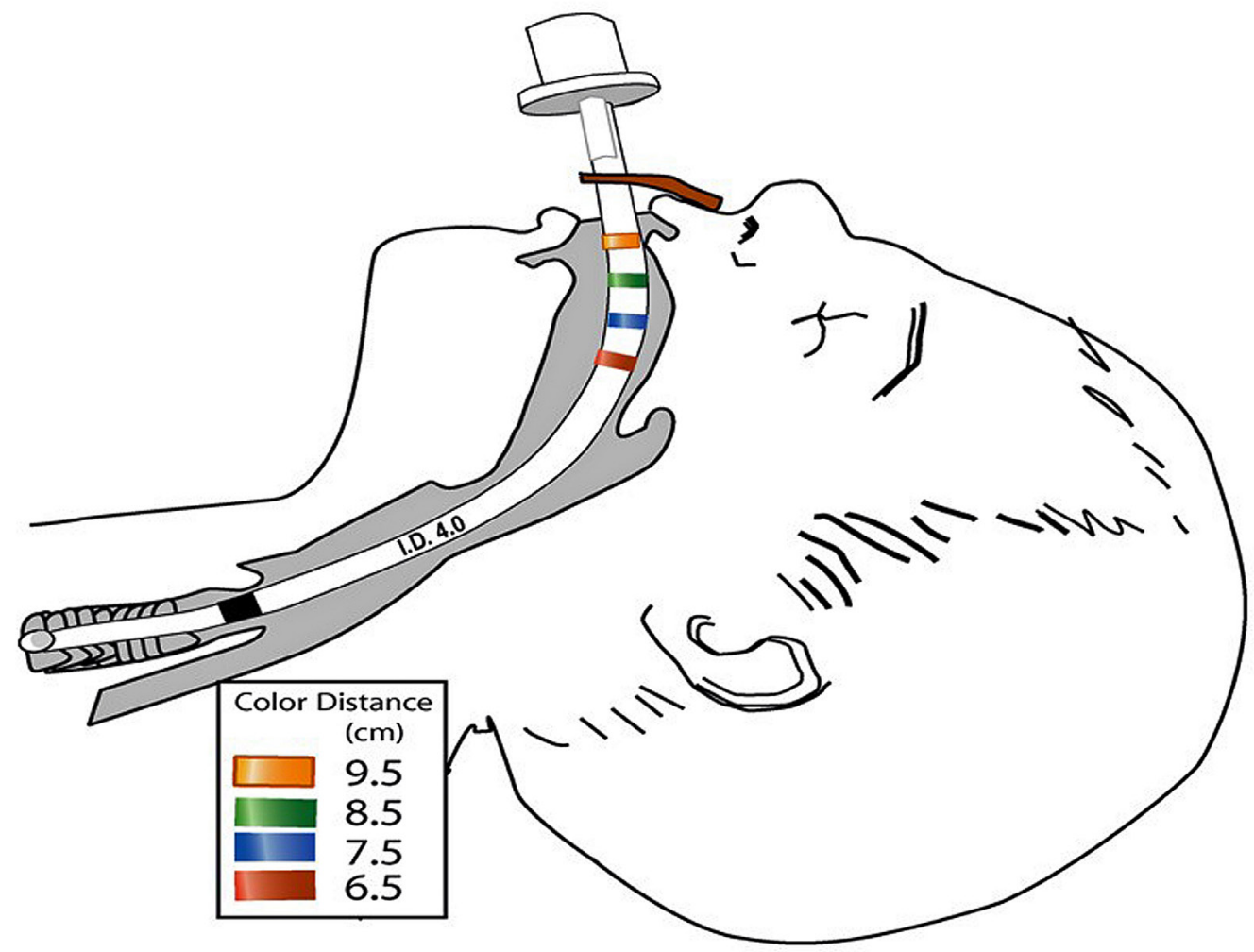

Figure 1: Representation of a new endotracheal tube design. We introduced the new tube into the trachea, and one of its proximally placed colored lines is next to the upper gingiva. We based the distance of insertion on an infant's weight in kilograms and the color of the line designates the depth of insertion for the different weights:

$>4 \mathrm{~kg}$ infant has a $9.5 \mathrm{~cm}$ distance of insertion and the colored line is "ORANGE",

$>3 \mathrm{~kg}$ infant has a $8.5 \mathrm{~cm}$ distance of insertion and the line is colored "GREEN"

$>2 \mathrm{~kg}$ infant has a $7.5 \mathrm{~cm}$ distance of insertion the line is colored "BLUE"

$>1 \mathrm{~kg}$ infants has a distance of insertion of $6.5 \mathrm{~cm}$ and the line is colored "RED".

We measured the depth of insertion from the upper gingiva to the tip of the endotracheal tube, and at this distance, the tip of the tube should be located to the mid-trachea.

\section{Methods}

\section{Retrospective Study}

A retrospective study reviewed the incidence of RMSBI before a prospective study using the newly marked endotracheal tube began. The review studied neonates hospitalized at the University of California, Davis Medical Center over a 3-year period. Our neonatal intensive care unit (NICU) identified all intubated infants. We reviewed charts of these infants for demographic information and reports of all chest radiographs.

Our protocol used in the NICU for performing chest radiographs keeps the head in the midline position with each arm extended beside the head. During the 3-year period, we used an ETT that had marks distally for localizing placement adjacent the vocal cords [1] and numbers in centimeters on the tube to determine the depth of insertion. The numbers in centimeters became higher as the distance from the ETT tip to the adaptor increases [7]. Information from the chart review established the incidence of RMSBI during the initial intubation and during the period of subsequent assisted ventilation. We correlated birth weight, gestational age, gender, and the duration of assisted ventilation with RMSBI.

\section{Prospective Study}

The second phase of this study enrolled 62 infants at University of California, Davis Medical Center at Sacramento, California. We estimated the sample size per the recommendations of Cohen for a chi-square analysis [8]. Using an effect size of 0.50 , a power of $.80, \mathrm{u}=1$, and an alpha of 0.05 , thirty-one infants were necessary for the study.

Inclusion criteria consisted of newborn infants regardless of birth weight and gestational age who required a) oro-tracheal intubation and b) infants who had parents who gave written informed consent for the prospective study. The exclusion criteria for newborn infants included babies with head, neck, or thoracic anomalies. Both groups received "State of the Art" neonatal care. The institutional review board of the University of California, Davis School of Medicine approved the retrospective study and prospective clinical trial of the ETT invention. 
A researcher obtained Informed consent from parents for use of the new ETT or the conventional ETT before birth. After written consent, the investigators randomized the participant to an ETT type that caregivers would use throughout the hospital stay. This protocol allowed use of a color-striped ETT or a conventional ETT for the initial endotracheal intubation. The investigators approached parents based on anticipated preterm birth or a fetal state that would likely require intubation after birth. Hence, the investigators obtained more written informed consents than the number of infants studied during the clinical trial of the new ETT design.

During the prospective study, we randomized infants to one of two groups based on the type of neonatal ETT used. We designated the control group be assigned to a group intubated with the same ETT used during the retrospective study. The ETT in the control group had a) circumferential stripes near the distal end that were used for vocal cord localization and b) centimeter numbers along its side of the tube to inform caregivers the distance from the upper lip, or the point of tube fixation, to the ETT tip in the trachea. The control and treatment ETTs had a Murphy eye that allowed potential ventilation of the left lung during RMSBI. The Murphy eye can hinder assessment of ETT location because bilateral breath and apical chest rise can still be present when there is RMSBI because of collateral ventilation.

The treatment group used the same ETT as control infants except the tubes had colored lines placed circumferentially at 6.5, 7.5, 8.5 and 9.5 centimeters from the tip (Figure 1). After oro-tracheal intubation, the treatment group had one colored line on the ETT placed adjacent to the midline of the upper gingiva. In this study, the experimental ETT had a non-toxic and tightly adherent tape that was $0.25 \mathrm{~cm}$ wide placed at the above-mentioned distances from the tip. Tubes with the adherent tape were prepared and gas sterilized before use.

Doctors Hipolito, Milstein and Sherman educated the respiratory care practitioners, NICU nurses, resident physicians, and neonatal fellows about the new ETT design and its appropriate use and placement before the study began in the NICU. Education of caregivers was ongoing during the prospective study.

Caregivers used the oro-tracheal intubation procedure described in the Textbook of Neonatal Resuscitation, American Academy of Pediatrics, $6^{\text {th }}$ Edition, 2011, Elk Grove, IL, for control and treated infants. Table 1 reviews the rule used to place ETT in control infants. The rule uses a 7,8, or $9 \mathrm{~cm}$ depth of oro-tracheal insertion from lip to the tip when the birth weight is 1,2 or 3 $\mathrm{kg}$, respectively $[2,9,10]$. The physician responsible for an individual infant decided the depth of insertion in centimeters based on either an estimated fetal weight or the actual birth weight. We based the estimated fetal weight on the average weight for the calculated gestational age of a fetus determined by either the last menstrual period or the fetal ultrasound examination. For control subjects, we based the depth of insertion on the centimeter marks imprinted on the tube. For endotracheal tubes with colored lines, we used the estimated fetal weight or actual birth weight to determine placement, however, we reduced the depth of insertion by $0.5 \mathrm{~cm}$ (Table 1). The reason for reducing the depth of insertion for ETTs with Colored Lines was one of the lines was located at the mid-point of the upper gingival ridge rather than locating a centimeter number to the mid-upper lip. To prevent movement of the ETT, we placed the Colored Line on an ETT at a fixed anatomic structure, the upper gingiva, rather than the more mobile upper lip, a traditional location for securing an ETT. Before the study, the researchers made measurements from the midlip to mid-upper gingiva in infants weighing from 500 to 4000 grams at birth. The mean distance of $\sim 0.5 \mathrm{~cm}$ over this birth weight range. For all infants who needed an emergent intubation over this range of birth weights subtracting a $0.5 \mathrm{~cm}$ distance from the 7-8-9 rule seemed appropriate during use of the new ETT design.

\begin{tabular}{|c|c|c|}
\hline \multirow[t]{2}{*}{ Weight, kg } & \multicolumn{2}{|c|}{ Mid-Line Depth of Oro-tracheal Tube Insertion } \\
\hline & Centimeters from upper lip ${ }^{*}$ & Centimeters from upper gum line \\
\hline $1 \dagger$ & 7 & 6.5 \\
\hline 2 & 8 & 7.5 \\
\hline 3 & 9 & 8.5 \\
\hline 4 & 10 & 9.5 \\
\hline \multicolumn{3}{|c|}{$\begin{array}{l}{ }^{\star} \text { Textbook of Neonatal Resuscitation }[2] \\
\dagger \text { Babies weighing }<750 \mathrm{~g} \text { require a } 6 \text { centimeter depth of insertion from ti } \\
\text { Caregivers consider this recommendation when inserting an emergency } \\
\text { endotracheal tube into infants below this weight. }\end{array}$} \\
\hline
\end{tabular}

Following insertion, we secured the ETT in control and treatment group infants to the upper lip using a described method [11]. Briefly, we applied a protective skin agent on the upper lip (No Sting Skin Prep ${ }^{\text {mi }}$ [Smith \& Nephew, Memphis, TN]). Next, we exactly cut an Elastoplast bandage (Tensoplast ${ }^{\mathrm{TM}}$ elastic adhesive bandage, BSM Medical, Charlotte, NC) and applied this bandage to the upper lip from one corner of the mouth to the other side. In the middle of the Elastoplast bandage, we placed a $0.25 \mathrm{~cm}$ central fold. We passed a 4-0 silk suture with a cutting needle through the fold in the Elastoplast and then passed the needle through the wall of the ETT taking care to avoid the lumen (4-0 black silk Ethicon suture 18", J-1 cutting). Two square knots secured the ETT to the Elastoplast bandage. Thereafter, we cut white adhesive tape into an H-shape. We secured the upper half of the white " $\mathrm{H}$ " adhesive tape to the Elastoplast bandage, while we wrapped the lower half of the " $\mathrm{H}$ " circumferentially around the ETT. 
We then covered the white adhesive tape with soft silicone tape (Mepitac silicone tape, Mölnlycke Health Care, Brunswick, ME) from either side of the cheek, over the lip and around the ETT. This pink silicone tape avoided oral secretions from wetting and loosening the white adhesive tape. We selected this technique for stabilizing the ETT because our NICU had 30 years of experience with the method. Moreover, we used this procedure for securing ETTs in the retrospective study.

After intubation in the delivery suite or after re-intubation in the NICU, we secured an ETT with an "H"-shaped adhesive tape to the upper lip and we reinforced the white adhesive tape with the pink silicone soft tape. Thereafter, a chest radiograph identified the location of the ETT tip. Then we applied the permanent method of stabilizing the ETT that uses the Elastoplast with suturing to the ETT.

Following oro-tracheal intubation, the initial and all subsequent chest radiographs were performed with the ETT in a midline position and the neck located in a neutral position without either flexion, extension, or rotation because such movements can change the position of the ETT in the trachea [12]. We adjusted an ETT to a mid- tracheal position after the radiograph. Subsequently, we performed follow-up chest radiographs depending on the clinical status and the discretion of the attending neonatologist. We obtained a chest radiograph if any respiratory examination had findings suspicious for RMSBI. Lastly, a pediatric radiologist unaware of the assignment to the treatment or control interpreted the ETT location independently.

During the course of assisted ventilation, the nursing staff, the respiratory care practitioners, or physicians assessed the proper location of the endotracheal tube by looking at the centimeter numbers on the ETT in control infants or by inspecting placement of the colored line next to the upper gingival ridge in treated infants.

For demographics, we recorded the following characteristics in both groups: birth weight, gestational age, gender, and the position of the endotracheal tube on chest radiograph.

\section{Statistical Analysis}

We stored clinical information and performed statistical analyses using SPSS version 22 (IBM, Chicago, Illinois). We conducted an inferential analysis using the Student's t test and a one-sample Chi-square test based on the data being analyzed [13]. Bonferroni correction was applied for comparison of the multiple groups in the retrospective study with the level of significance set at $P=.01$.

\section{Results}

\section{Retrospective Study}

During a 3-year period, 447 neonates required intubation during hospitalization. Table 2 shows the demographic findings. The overall incidence of RMSBI was 20\%; however, Figure 2 shows that infants born at 23 - 26 weeks of gestation had a $57 \%$ incidence of bronchial intubation either right after the initial intubation or during the course of assisted ventilation $\left(\chi^{2}=54.93, P<.001\right)$. When infants born at 23 - 26 and 27 - 29 weeks of gestation were compared to infants born at $\geq 37$ weeks of gestation, there was a higher risk of RMSBI at the younger gestational age $\left(\chi^{2}=49.27, P<.001\right.$ and $\chi^{2}=6.81, P<.01$, respectively). An analysis of chest radiographs during assisted ventilation revealed that a longer period of ventilatory support increased the risk of RMSBI. Infants born between 23 - 26 weeks of gestation had the longest duration of ventilator care.

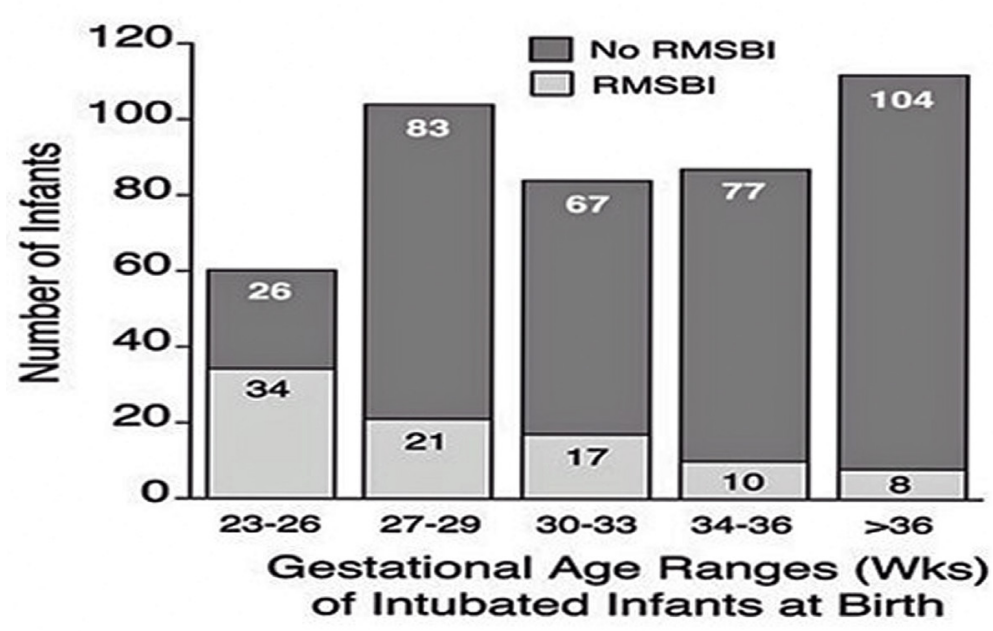

\footnotetext{
Figure 2: Relationship between gestational age and the incidence of bronchial intubation in neonates. This figure shows the gestational age at birth (in the weeks after conception) on the " $x$ " axis, and we display the number of subjects that did or did not have bronchial intubation on the "y" axis. The internal legend shows infants in each gestational age category that did have (light-colored fill) or did not have (darkcolored fill) right main stem bronchial intubation. The inverse relationship between advancing gestational age and a reduction in the risk of bronchial intubation is evident. Right main stem bronchial intubation is abbreviated RMSBI.
} 


\begin{tabular}{|c|c|}
\hline Gestational Age in Weeks of the Infants $^{*}$ & Number of Intubated Infants $^{*}$ \\
\hline $23-26$ & 60 \\
\hline $27-29$ & 104 \\
\hline $30-33$ & 84 \\
\hline $34-36$ & 87 \\
\hline $37-42$ & 112 \\
\hline Gender & 237 \\
\hline Male & 210 \\
\hline Female & \\
\hline${ }^{*}$ Birth weights ranged from 455 to 4185 grams. & \\
\hline
\end{tabular}

Table 2: Demographic Findings in the Retrospective Study

\section{Prospective Study}

Table 3 shows the demographic findings of subjects in the prospective study. There was no statistical difference in birth weight, gestational age, or gender between the treatment and control groups. Levene's Test for the Equality of Error Variances was not significant $(P>.05)$. We assumed equal variances in control and treated groups.

\begin{tabular}{|c|c|c|c|c|}
\hline \multirow{2}{*}{ Range Gestational Age (weeks) } & \multicolumn{2}{|c|}{ No Colored Line $(\mathbf{n}=\mathbf{3 1})$} & \multicolumn{2}{c|}{ Colored Lines $(\mathbf{n}=\mathbf{3 1})$} \\
\cline { 2 - 5 } & $\mathbf{n}$ & $(\mathbf{\%}$ of $\mathbf{n})$ & $\mathbf{n}$ & $(\mathbf{\%}$ of $\mathbf{n})$ \\
\hline $23-26$ & 2 & 6 & 5 & 16 \\
\hline $27-29$ & 7 & 23 & 8 & 26 \\
\hline $30-33$ & 8 & 26 & 9 & 29 \\
\hline $34-36$ & 3 & 10 & 5 & 16 \\
\hline $37-\mathbf{4 2}$ & $\underline{11}$ & $\underline{35}$ & $\underline{4}$ & $\underline{13}$ \\
\hline $\begin{array}{c}\text { Median Days Intubated: } \\
\text { (Range) }\end{array}$ & 14 days & & 17 days & \\
\hline Gender & $(1-48 \mathrm{~d})$ & & $(2-55$ d $)$ & \\
\hline Male & 19 & 61 & 18 & 58 \\
\hline Female & 12 & 39 & 13 & 42 \\
\hline
\end{tabular}

Table 3: Demographics of Infants in the Control Group (Endotracheal Tubes with commercial markings) and the Treatment Group (Endotracheal Tubes with Proximally Placed Colored lines)

In the control group, 8 of $31(26 \%)$ of the subjects had RMSBI either after an initial ETT placement $(\mathrm{n}=3)$ or during subsequent ventilator care $(n=5)$. None of the 31 infants who had a color stripped ETT placed experienced RMSBI either initial or subsequent RMSBI during ventilator care on chest radiography $\left(x^{2}=34.13, P<.001\right)$. Additionally, two of 31 infants in the control group (conventional ETT) and none of 31 infants in the treated group (color-striped ETT) experienced an unanticipated or unscheduled extubation. After the initial stabilization of the ETT in the mid-trachea until the time of elective extubation, the number of chest radiographs were not statistically different between the treated and control subjects. The researchers observed no additional adverse outcomes with the new ETT design.

\section{Discussion}

Kuhns and Poznanski first proposed that manufacturers place centimeter numbers on ETTs with the higher numbers indicating a greater distance from the tip of the tube [1]. These numbers gave rise to the 7-8-9 rule that relates to the depth of insertion of an oro-tracheal ETT in a neonate of a specific weight $[2,9,10]$. Loew and Thibeault described an alternative method to control the depth of insertion during intubation [7]. A black safety line is located proximal to the tip of the ETT. This black line is the vocal cord localizer. The black line is safety strategy wherein this line placed at the vocal cords prevents RMSBI when tubes are inserted using direct vision and laryngoscopy. A report, however, suggests vocal cord localizers on ETTs do not always accurately localize the tube in a proper position [14]. Our retrospective review showed that intubations still carry an unacceptable risk of bronchial intubation with an occurrence of approximately $26 \%$. We observed an incidence of $26 \%$ RMSBI in the control group during the prospective randomized clinical trial of the new ETT design. Twenty-six percent is lower than the $50 \%$ incidence reported in 1971 [1]. Today, this occurrence of RMSBI is not acceptable because more ELBW infants are born and caregivers and parents anticipate an intact survival. In immature infants, RMSBI is still occurring and pulmonary morbidity and mortality are serious consequences $[15,16]$. 
Following intubation, and during the process of externally securing an ETT, the caregiver can no longer see the vocal cord localizer. Physicians appreciate an ETT may become poorly positioned during fixation. The back-up strategy for the vocal cord localizer is the numbers displayed in centimeters on side of the tube. The centimeter numbers are small and taping the tube to the lip or a stabilizing device obscures the numbers from view. Currently, manufacturers are selling a number of fixation devices to secure the ETT rather than using the older method of taping the ETT to the upper lip and face. Nevertheless, caregivers still use taping of the ETT to the lip until a chest radiograph defines the ETT location and an adjustment of the ETT tip to a mid-trachea is undertaken. ETT-related fixation devices can still create problems in identifying the centimeter numbers imprinted on an ETT. A study found an ETT stabilizing device prevented low placement compared to a taping method; however, this device still resulted in an unsatisfactory localization of ETTs in the trachea of infants [17]. If caregivers secure an ETT to the upper lip with tape, there may be substantial inward and/or outward movement of the tube. We theorized we could readily see colored, circumferential lines placed proximately on ETTs rather than struggling to see tiny numbers on the tube. We proved our hypothesis was correct if a colored line on an ETT was adjacent to a fixed anatomic structure like the upper gingiva. We could easily see the colored lines on an ETT by simply lifting up the lip and looking into the mouth. This examination assured the caregiver that the original location of the ETT had not changed.

We used colored lines placed on ETTs since they have a relationship to the 7-8-9 rule $[2,9,10]$. By localizing a colored line on the ETT to the upper gingiva rather than the lip, we had to subtract $0.5 \mathrm{~cm}$ from the $7-8-9$ rule. The bright colored lines conform to colors used on signal flags or traffic signs. Our study found the colored lines on ETTs located at the upper gingival ridge prevents RMSBI during the initial intubation and subsequent ventilator care. Nurses and respiratory practitioners reported the colored lines readily identified the depth of insertion compared to the small centimeter numbers affixed to ETTs.

Researchers reported infants weighing $<750 \mathrm{~g}$ at birth do not conform to the 7-8-9 rule [10]. The authors suggested caregivers place an ETT at $5.5 \mathrm{~cm}$ at the lip if an infant is born between 22 to 24 weeks of gestation or has a birth weight between 400 to 600 grams. A separate study found that gestational age, rather than birth weight, had a linear relationship with the proper depth of ETT-related insertion [18]. Researchers must consider these reports when performing future studies of ETT placement and when assessing whether proximal colored lines on ETTs can prevent RMSBI in micropreemies.

This study has limitations. We could not mask the colored lines on the ETT from the caregivers who knew immediately the assignment to the control or treatment group. It is conceivable that bedside nurses, respiratory care practitioners, and physicians were more attentive to displacement of an ETT with colored lines. Alternatively, these caregivers may have been more attentive to the participants without a color-striped ETT because of a perceived increased risk of dislocation. This is particularly true because of education that NICU-related caregivers received from investigators before beginning the prospective clinical trial. Since the Hawthorne effect, in which information available to the caregivers potentially influences their behavior, there is a potential threat to the validity of this study. Nevertheless, the endotracheal tube design and its method of localization are defensible concepts.

Additionally, inward displacement of ETT in neonates carries a risk of RMSBI when caregivers secure ETTs by taping to the lip and face. We redesigned the markings on ETTs because many NICUs worldwide still secure ETTs by taping initially after intubation and also during ventilator care because of convenience or the high cost of ETT attachment devices. Furthermore, many attachment devices are excessively large for extremely preterm infants. Yet, it is conceivable that one of the newer devices to secure ETTs might reduce RMSBI in larger neonates during assisted ventilation. However, more stable attachment devices that secure ETTs can still move. It is noteworthy that the Food and Drug Administration in the United States has approved new devices for the fixation of ETTs in neonates without requiring manufacturers to perform safety studies showing lower rates of RMSBI when caregivers use of the appliance. Additional clinical trials comparing the merits of this new ETT design versus the benefits of new fixation devices in preventing bronchial intubation during assisted ventilation of neonates seem warranted.

\section{Conclusion}

In the future, we propose use of neonatal ETTs with bright, circumferential colored lines placed proximally on the tube. These lines should correspond to a distance to the tip of the tube. During oro-tracheal intubation, we have recommended the colored lines reside next to the mid-upper gingiva, a stable anatomic structure that cannot move like the upper lip. This initial report suggests this innovation in endotracheal tube design is safe and lowers the risk of bronchial intubation. Use of this endotracheal tube design may also reduce ventilator-related morbidity in ELBW infants that have the highest risk of bronchial intubation. The investigators suggest caregivers undertake additional clinical studies with the new ETT design before its routine use in neonatal care.

\section{Acknowledgment}

A grant from the Children's Miracle Network supported this research. We thank the respiratory care practitioners and nursing staff that provided care in the NICU at the University of California - Davis neonatal intensive care unit. We thank Robin White for maintenance of the database in our NICU and Stephen Bennett for preparation of the illustrations. 


\section{References}

1. Kuhn LR, Poznanski AK (1971) Endotracheal position in the infant. J Pediatr 78: 991-6.

2. Kattwinkel J (2002) Endotracheal Intubation. Textbook of Neonatal Resuscitation, (4th Edition). Am Acad Pediatr, Elk Grove Village, IL.

3. Thibeault DW, Lachman RS, Laul VR, Kwong MS (1973) Pulmonary interstitial emphysema, pneumomediastinum, and pneumothorax in the newborn infant. Am J Dis Child 126: 611-4.

4. Hill A, Perlman JM, Volpe JJ (1982) Relationship of pneumothorax to occurrence of intraventricular hemorrhage in the premature newborn. Pediatrics 69: 144-9.

5. Roopchand R, Roopnarinesingh S, Ramsewak S (1989) Instability of the tracheal tube in neonates. A postpartum study. Anaesthesia 44: 107-9.

6. Wilson A, Gardner MN, Armstrong MA, Folck BF, Escobar GJ (2000) Neonatal assisted ventilation: Predictors, frequency, and duration in a mature managed care organization. Pediatrics 105: 822-30.

7. Loew A, Thibeault DW (1974) A new and safe method to control the depth of endotracheal intubation in neonates. Pediatrics 54: 506-8.

8. Cohen J (1988) Statistical Power Analysis for the behavioral sciences. (2 ${ }^{\text {nd }}$ Edition), Hillsdale, New Jersey, Lawrence Erlbaum Associates.

9. Tochen ML (1979) Orotracheal intubation in the newborn infant: a method for determining depth of tube insertion. 95: 1050-1.

10. Peterson J, Johnson N, Deakins K, Wilson-Costello D, Jelovsek JE, et al. (2006) Accuracy of the 7-8-9 Rule for endotracheal tube placement in the neonate. J Perinatol 26: 333-6.

11. Gregory GA (1972) Respiratory care of newborn infants. Pediatr Clin North Am 19: 311-24.

12. Donn SM, Kuhns LR (1980) Mechanism of endotracheal tube movement with change of head position in the neonate. Pediatr Radiol 9: 37-40.

13. Glantz SA (2012) Primer of Biostatistics, (7 $7^{\text {th }}$ Edition). McGraw-Hill, New Year.

14. Molendijk H (2001) Use of the black area on the tube tip for rapid estimation of insertional depth of endotracheal tubes in neonates: a potential hazard. Arch Dis Child 85: F77.

15. Williams DW, Merten DF, Effmann EL, Scatliff JH (1988) Ventilator-induced pulmonary pseudocysts in preterm neonates. AJR Am J Roentgenol 150: 885-7.

16. Thayyil S, Nagakumar P, Gowers H, Sinha A (2008) Optimal endotracheal tube tip position in extremely premature infants. Am J Perinatol 25: 13-6.

17. Brinsmead TL, Davies MW (2010) Securing endotracheal tubes: does NeoBar availability improve tube position? J Paediatr Child Health 46: 243-8.

18. Kempley ST, Moreiras JW, Petrone FL (2008) Endotracheal tube length for neonatal intubation. Resuscitation 77: $369-73$.

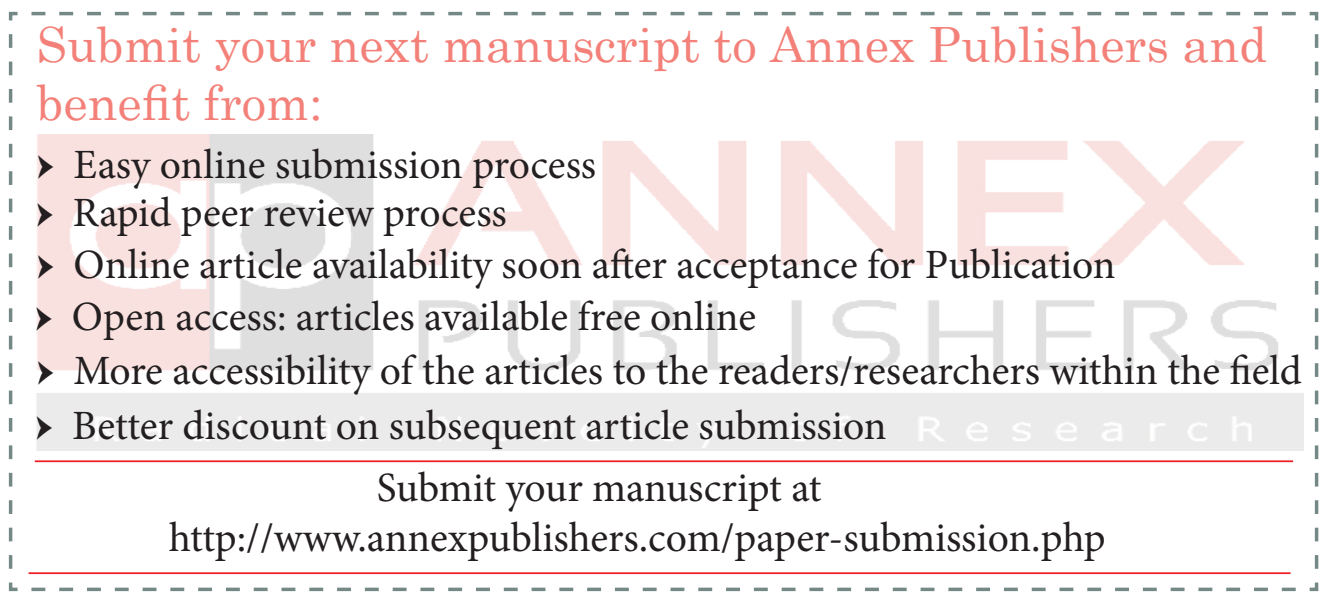

